\title{
EMPTIO IACTUS MISSILIUM
}

\author{
FRANCISCO BARTOL
}

UNED

Resumen: Durante el Principado los emperadores romanos acostumbraban, principalmente al ir o al salir de los juegos, a lanzar objetos en ocasiones muy valiosos (iactus missilia). Había ciertas personas que previamente compraban a los expertos recogedores sus posibles capturas, ya que éstos se convertían en propietarios de las res derelictae. Los juristas romanos que equipararon esta emptio-venditio con el captum piscium vel avium, la calificaron como emptio aleae aut spei.

Palabras clave: iactus missilia, res derelictae, alea, spes, emptio iactus missilium, iactus retis, emptio spei hereditatis, aucupium, piscatio.

Abstract: In the Principality, when going to or returning from the circus games, the roman emperors principally used to throw objects which were sometimes very valuable (iactus missilia). There were certain people who bought previously the possible collection of the expert pickers, as they became the owners of the res derelictae. The roman jurists who considered equally this emptio-venditio with the captum piscium vel avium qualified the first one as emptio aleae aut spei.

Keywords: iactus missilia, res derelictae, alea, spes, emptio iactus missilium, iactus retis, emptio spei hereditatis, aucupium, piscatio. 


\section{ORIGEN Y NATURALEZA DEL IACTUS MISSILIUM}

La romanística ${ }^{1}$ con fundamento en el texto ciceroniano: Tuditanus nempe ille, qui cum palla et cothurnis nummos populo de rostris spargere solebat ${ }^{2}$, ha estimado que los iactus missilium tenían sus inicios en la República. Es posible que en el s. I a.C. hubiera algún caso aislado como el de Tuditano, al que tanto Cicerón ${ }^{3}$ como Valerio Máximo $^{4}$ consideran literalmente un loco, sin embargo, en nuestra opinión, la costumbre de spargere missilia se inició en los primeros años del Principado.

Los primeros emperadores romanos, debido a su política populista, acostumbraban, sobre todo cuando acudían o salían de la celebración de los ludi gladiatorii o scaenici, a arrojar al pueblo diversos objetos denominados globalmente missilia ${ }^{5}$ para demostrar así su generosidad. La prerrogativa perteneció también durante esta época a los ciudadanos ricos. Justiniano en sus Instituciones sustituye a estos nobiles por los pretores y los cónsules ${ }^{6}$.

Etimológicamente el término missilia significa lo lanzado, lo enviado. Este vocablo que es propio del lenguaje militar, tal como puede apreciarse en los historiadores romanos, Tito Livio $^{7}$, Tácito ${ }^{8}$ o Amiano Marcelino ${ }^{9}$, generalmente aparece unido al término telum. Así Festo escribe, Tela proprie dici videntur ea, quae missilia sunt, ex Graeco videlicet translato eorum nomine, quoniam illi thlOqen missa dicunt, quae nos eminus ${ }^{10}$.

1 CALONGE, La compraventa civil de cosa futura, Salamanca 1963, 34.

2 Cic., In M. An. 3,16.

${ }^{3}$ Cic., Academica priora sive luculus, 89, Quid loquar de insanis: qualis tandem fuit adfinis tuus Catule Tuditanus, quisquam sanissimus tam certa putat quae videt quam is putabat quae videbantur?

${ }^{4}$ Val. Max. 7,8,1, Quam certae, quam etiam notae insaniae Tuditanus! utpote qui populo nummos sparserit togamque uelut tragicam uestem in foro trahens maximo cum hominum risu conspectus fuerit ac multa his consentanea fecerit.

5 FERNÁNDEZ DE BUJÁN, F., Sistema contractual romano, Madrid 2003, $172-$ 173.

${ }^{6}$ IJ.2,1,46.

7 Tit. Liu, $A b$ urbe condita, 23, 19,5; 27,15,5; 30,10,7;38,19,6;38,22,8; 42,53,4; $44,35,21$.

8 Tac. De uita Iulii Agricolae, 36,1; Germania, 6,1; Historiae, 3,71,2; 4,71,5.

9 Ami. Mar. 14,2,18; 20,11,21; 24,5,2; 31,6,3.

10 Fest. De uerborum significatu, 502. Esta misma definición aparece en Aulo Gellio, Noct. Att., 9,1,3, Tum Iulianus comprobato genere quaestionis: "quod de sagitta" inquit «et lapide dixit, hoc de omni fere missili telo dici potest. Para Gayo en el 2 ad leg. XII Tab. (D.50,16,233,2) el término telum se refiere también a lo que es lanzado con la mano. 
El objeto de estos missilia, en palabras de Suetonio, era muy variado: Augusto y Calígula arrojaron todo tipo de víveres, frutas ${ }^{11}$ y monedas $^{12}$, y Nerón lanzó aves, bonos para el trigo, vestidos, oro, plata, piedras preciosas, cuadros, e, incluso, cédulas canjeables por esclavos, por bestias, por fieras domesticadas, por navíos, por bloques de casas, o por campos ${ }^{13}$.

Los términos para expresar este lanzamiento fueron distintos, Suetonio siempre utiliza sparsio, en cambio, Gayo prefiere iactatio ${ }^{14}$. Su significación es muy diferente, iactatio se emplea cuando se requiere un movimiento brusco del brazo, por eso en las fuentes literarias se usa con lapides o tela; sparsio, por el contrario, suele referirse a algo menos pesado como pueden ser los nummos ${ }^{15}$ o los semina ${ }^{16}$.

Una Constitución de los emperadores Valentiniano y Marciano del año 452 considera una vileza y un error que los cónsules arrojen monedas al pueblo, y aconseja que con este dinero se hagan obras provechosas para la ciudad:

Cessante ergo ista spargendi vilitate amplissimi consules procedentes deinceps abstineant hoc errore perdendi, optimoque consilio operi necessario proficiat, quod erat incompetenter proiciendum ${ }^{17}$.

Gayo en el libro segundo de las rerum cottidianarum sive aureorum (D. $41,1,9,7)$ escribe que cuando se arrojan cosas la voluntad del propietario transmite la propiedad y quien las recoge se convierte en propietario:

Hoc amplius interdum et in incertam personam collocata uoluntas domini transfert rei proprietatem: ut ecce qui missilia iactat in uulgus, ignorat enim, quid eorum quisque excepturus sit, et tamen quia uult quod quisque exceperit eius esse, statim eum dominum efficit.

11 Suet., Aug. 98,3: pomorum et obsoniorum rerumque missilia; Calig., 18,2, sparsit et missilia uariarum rerum et panaria cum obsonio uiritim diuisit.

12 Suet., 37,1: quin et nummos non mediocris summae e fastigio basilicae Iuliae per aliquot dies sparsit in plebem.

${ }_{13}$ Suet., Nero, 11,2: sparsa et populo missilia omnium rerum per omnes dies: singula cotidie milia auium cuiusque generis, multiplex penus, tesserae frumentariae, uestis, aurum, argentum, gemmae, margaritae, tabulae pictae, mancipia, iumenta atque etiam mansuetae ferae, nouissime naues, insulae, agri.

${ }^{14}$ La jurisprudencia emplea el término iactus en lugar de iactatio, cuando hace referencia a la Lex Rhodia de iactu: D.14.2.0., por ejemplo Paulo en D.14,2,2: Si laborante nave iactus factus est; Juliano en D.41,7,7, Si quis merces ex nave iactatas invenisset...

${ }_{15}$ D.18,1,8,1: Cum quis aes sparserit... CJ.12,3: De consulibus et non spargendis ab his pecuniis...

16 Sen. Epist. 90,21; Quint. Inst. 2,9,3.

17 CJ. 12,3,2,2. 
Justiniano en las Instituciones 2,1,46, reproduce este mismo texto:

Hoc amplius interdum et in incertam personam collocata voluntas domini transfert rei proprietatem: ut ecce praetores vel consules qui missilia iactant in vulgus ignorant quid eorum quisque excepturus sit, et tamen, quia volunt quod quisque exceperit eius esse, statim eum dominum efficiunt.

No existen dudas de que el texto justinianeo fue tomado del gayano, sin embargo, hay unas diferencias textuales que es necesario indicar. Mientras para Gayo el sujeto, es decir, la persona que lanza los missilia, es qui (quien, el que) y, en consecuencia, indeterminada, en cambio, el emperador determina claramente los sujetos, praetores vel consules. Esta modificación textual implica necesariamente variaciones en las personas verbales (iactat-iactant; ignorat-ignorant; vult-volunt; efficit-efficiunt $)^{18}$. La segunda disparidad textual está en la conjunción enim; su presencia en el texto postclásico nos induce a pensar que tiene al mismo tiempo un significado explicativo y otro acumulativo, además o también ${ }^{19}$.

En el iactus missilium está, por una parte, la voluntad del dueño de abandonar estas res missiles, que se convierten en res derelictae, y transferir la propiedad in incertam personam ${ }^{20}$, y por otra, la voluntad del primer ocupante de adquirirlas. Pomponio poniendo como ejemplo el aes spargere escribe que, cuando se abandona una cosa, se tiene la voluntad de que sea de otro:

D.41,7,5,1 (Pomponius libro trigensimo secundo ad sabinum)

Id, quod quis pro derelicto habuerit, continuo meum fit: sicuti cum quis aes sparserit aut aves amiserit, quamvis incertae personae voluerit eas esse, tamen eius fierent, cui casus tulerit ea, quae, cum quis pro derelicto habeat, simul intellegitur voluisse alicuius fieri.

A. d'Ors considera este iactus un tipo especial de derelictio. Se trata en época clásica de una traditio donationis causa a una persona indeterminada ${ }^{21}$. La adquisición derivativa, traditio ad incertam personam, surge de la correspondencia existente entre el acto de abandonar y el de ocupar, puesto que puede ser necesaria la usucapión $(\text { pro derelicto })^{22}$.

\footnotetext{
cribe ad incertam personam.

21 D'ORS, A., Derecho Privado Romano ${ }^{8}$, cit., 164, n. 4.

22 D'ORS, A., Derecho Privado Romano ${ }^{8}$, cit., 158.
}

18 BENEDEK, Iactus missilium, en Sodalitas a Guarino 5, (1984), 2111.

19 BENEDEK, en Iactus missilium, cit., 2111, sostiene que la omisión de este término indica una relación más estrecha con la segunda parte del texto.

20 D'ORS, A., Derecho Privado Romano ${ }^{8}$, Navarra 1991, 164, n. 4, conocedor del sentido de hostilidad que tenía la preposición in con acusativo en época clásica es- 
En las Instituciones justinianeas $(2,1,47)$ hay una insertio, en palabras mommsenianas ${ }^{23}$, explicativa de la adquisición de la propiedad de las res missiles:

Qua ratione verius esse videtur et si rem pro derelicto a domino habitam occupaverit quis, statim eum dominium effici. pro derelicto autem habetur quod dominus ea mente abiecerit ut id rerum suaram esse nollet, ideoque statim dominus esse desinit.

\section{EMPTIO IACTUS MISSILIUM}

La problemática más importante del iactus missilium se plantea con relación a la compraventa. Pomponio considera la emptio missilium como una emptio aleae aut spei, al equiparar ambos términos:

\section{D.18,1,8,1 (Pomponius libro nono ad Sabinum)}

Aliquando tamen et sine re venditio intellegitur, veluti cum quasi alea emitur. quod fit, cum captum ${ }^{24}$ piscium vel avium ${ }^{25}$ vel missilium emitur: emptio enim contrahitur etiam si nihil inciderit, quia spei emptio est: et quod missilium nomine eo casu captum est si evictum fuerit, nulla eo nomine ex empto obligatio contrahitur, quia id actum intellegitur.

Aunque etimológicamente los términos spes y sperata tienen la misma raíz $z^{26}$ y en consecuencia un significado semejante, sin embargo, desde el punto de vista jurídico la diferencia es clara: en la emptio rei speratae la compraventa está condicionada por la existencia de la cosa futura, en cambio, la emptio spei no presenta ninguna condición, porque lo que se compra es el alea.

23 MOMMSEN, i.a.c. Editio Maior, vol. II, 491.

24 Como dice ARANGIO-RUIZ, en La compravendita in diritto romano, vol. I, Napoli 1956 , p. 119, es un término corrupto por captus. No puede ser un acusativo singular porque el verbo está en forma pasiva.

${ }_{25}$ Tip. leen apium, ya que han transcrito melissîn.

${ }^{26}$ Enn. en Annales 128, emplea speres como plural de spes. Relacionados con este término están desperatus (sin esperanza) y prosperus (con esperanza). 
Alea ${ }^{27}$ se refiere generalmente al juego de los dados, muy practicado por los romanos ${ }^{28}$, y, según Cicerón, nada hay más incierto que el resultado de la tirada de los éstos ${ }^{29}$. Este término latino, para Boselli, significa acontecimiento incierto, aunque comúnmente se emplea como sinónimo de riesgo. La diferencia está en que alea indica el estado de esperanza y temor al mismo tiempo, y riesgo expresa el aspecto negativo de esa incertidumbre, ya que suele tener el significado de peligro de un $\mathrm{mal}^{30}$.

El término spes significa que se espera un resultado positivo. Se habla siempre de emptio spei, porque es el comprador quien necesariamente tiene que tener una bona spes. Ahora bien, la compra de la spes, es como nos dice Pomponio, la compra del azar (alea) aunque el riesgo no sea igual en todos los supuestos ${ }^{31}$.

En opinión de Longo la emptio spei es una irregularidad del contrato de compraventa ${ }^{32}$, y Zimmermann se pregunta sí este tipo de transacciones no son demasiado simples como para merecer un análisis jurídico ${ }^{33}$.

27 San Isidoro, en su afán de dar una explicación racional a todos los términos latinos, piensa que el juego fue inventado por un soldado llamado Alea, durante la guerra de Troya, Etym. XVIII,60: Alea, id est lusus tabulae, inventa a Graecis in otio Troiani belli a quodam milite Alea nomine, a quo et ars nomen accepit. Tabula luditur pyrgo, calculis tesserisque. La referencia más antigua que tenemos sobre una ley del juego es de Plauto en Mil. Glo. 164-165:

atque adeo ut ne legi fraudem faciant aleariae,

adcuratote ut sine talis domi agitent convivium.

El juego de los dados es citado también por Horacio, Car., III,24: seu malis uetita legibus alea. Ovidio en Trist. 2,471: Sunt aliis scriptae, quibus alea luditur, artes. Cicerón en In $M$. Ant. 2,23,56, afirma que Licinio Dentículo fue condenado por el juego: Licinium Denticulum de alea condemnatum.

${ }^{28}$ El término alea no indica el dado, sino el acto de echar los dados. La ocasión propicia para este juego eran los banquetes.

${ }_{29}$ Cic. De diuinatione, 2, 121: quid est tam incertum quam talorum iactus? El Digesto dedica un título a los jugadores de azar De aleatoribus (D.11,5,0). Al juego de azar también se refiere Pomponio en el libro 12 Ad Sabinum (D.17,2,59,1), y Escévola en el $l$. VI responsorum (D.22,2,5pr.). El juego fue prohibido por el emperador Justiniano CJ.3,43,1: ut nulli liceat in privatis seu publicis locis ludere neque in specie neque in genere.

30 BOSELLI, s.v. Alea, en NNDI, Torino, 1957, 469.

31 ARANGIO-RUIZ, compara la emptio spei con ciertos juegos de cartas actuales y la quiniela futbolística, La compravendita in diritto romano, cit., 121 n.1.

32 LONGO, Corso di diritto romano, Milán 1946, 349.

33 ZIMMERMANN, The law of obligations, Roman Foundations of the civilian tradiciton, Oxford 1996, 247. 
El jurista no menciona las venationes, cuando en opinión de Cicerón $^{34}$ era una dedicación muy frecuente entre los romanos en los ratos perdidos y, además, una parte de los juegos circenses, a los que solían acudir los emperadores y esparcir los missilia tal como nos comenta Suetonio. El aucupium y la piscatio exigen mucha astucia, habilidad y rapidez como nos dice Marcial o Silio Itálico ${ }^{35}$, en cambio, la venatio, requiere más fuerza y persecución.

Es posible que la emptio spei más antigua fuera la del iactus retis ${ }^{36}$. La pesca y la caza eran actividades muy frecuentes desde los primeros tiempos de la Monarquía y cuya finalidad era satisfacer una de las primeras necesidades del ser humano, alimentarse. El pedagogo Valerio Máximo ${ }^{37}$ nos expone el caso de unos pescadores que en Mileto habían vendido el iactum retis, y sacaron en lugar de peces un trípode de oro. Los pescadores afirmaban que ellos habían vendido solamente el pescado, el comprador, en cambio, decía que era todo el producto de sus redes. La asamblea del pueblo decidió que el caso fuera resuelto por el oráculo de Delfos, y éste estimó oportuno que el trípode fuera asignado al más sabio, con lo que el trípode terminó en manos del dios Apolo en Delfos.

${ }^{34}$ Cic. De Senectute, 56: Conditiora facit haec supervacaneis etiam operis aucupium atque venatio

35 Mart. 14,216: Non tantum calamis, sed cantu fallitur ales, Callida dum tacita crescit harundo manu

Sil. Ita., 5,47:

haud secus ac uitreas sollers piscator ad undas, ore leuem patulo texens de uimine nassam, cautius interiora ligat mediamque per aluum sensim fastigans compressa cacumina nectit ac fraude artati remeare foraminis arcet introitu facilem quem traxit $a b$ aequore piscem.

${ }^{36}$ DAUBE, Purchase of a peospective jul, en Studi Paoli (Florencia, 1955), cit., por CALONGE, p.35.

${ }^{37}$ Val. Max., 4,1,ext. 7: a piscatoribus in Milesia regione euerriculum trahentibus quidam iactum emerat. extracta deinde magni ponderis aurea Delphica mensa orta controuersia est, illis piscium se capturam uendidisse adfirmantibus, hoc fortunam ductus emisse dicente. qua cognitione propter nouitatem rei et magnitudinem pecuniae ad uniuersum ciuitatis eius populum delata placuit Apollinem Delphicum consuli cuinam adiudicari mensa deberet. deus respondit illi esse dandam, qui sapientia ceteros praestaret, his uerbis:

t...j sof...v pritoj pEntwn; toÚtJ tr...pod' aìdî.

tum Milesii consensu Thaleti mensam dederunt. ille cessit ea Bianti, Bias Pittaco, is protinus alii, deincepsque per omnium vii sapientium orbem ad ultimum ad Solonem peruenit, qui et titulum amplissimae prudentiae et praemium ad ipsum Apollinem transtulit. 
Es una emptio sine re, que es perfecta desde su origen. Guarino señala que la venta es válida, pero no es una verdadera venta, se trata más bien de una apuesta, bajo la forma de venta, es decir, un contrato aleatorio ${ }^{38}$.

En opinión de Calonge hay una clara diferencia entre la emptio spei propiamente dicha, que es la emptio aleae y cuyos supuestos son los iactus retis y missilium; y otras emptiones, donde el riesgo de azar no es pleno, como la emptio spei hereditatis o la emptio spei obligationis $^{39}$. Brasiello duda de la clasicidad de la emptio iactus missilium $^{40}$.

El emptor adquiría todo el producto alcanzado por un precio fijado anteriormente. Si no quería quedar defraudado requería por su parte la elección de una persona experta (peritus) en el arte de la pesca o caza de aves, o en conseguir los objetos lanzados. Cuanto más experto, mayor esperanza de obtener ganancia. Y el venditor, a pesar de conocer de antemano la merx, debía tener interés en obtener una gran cantidad de objetos, con la finalidad de que su fama le abriría las puertas a nuevas emptiones spei.

El texto pomponiano junto con este caso menciona la piscatio, y el aucupium. Y aunque hay una equivalencia, tal como indica el uso del mismo nexo disyuntivo vel, sin embargo, podemos encontrar algún matiz diferente que separaría por un lado la pesca y la caza de aves y, por otro, el lanzamiento de regalos ${ }^{41}$; en el primer caso el resultado no dependía solo de la experiencia del vendedor, intervenían también otras circunstancias que podían modificar dicho resultado, como las condiciones climáticas o la época.

La compra del iactus missilium era menos frecuente en Roma que la del captus piscium vel avium ${ }^{42}$. Para Beseler y Vasalli es un texto interpolado y, en consecuencia, debía eliminarse toda referencia a los missilia en este pasaje (D.18,1,8,1), es decir, a partir de et quod ${ }^{43}$. Ulpiano habla de la obligación del comprador de pagar el precio al pescador o al cazador aunque no consigan nada, pero, sin duda nin-

38 GUARINO, Diritto Privato romano, Napoli 1988, 785 ss.

39 CALONGE, La compraventa civil de cosa futura, cit. 33.

40 BRASIELLO, Emptio rei speratae et emptio spei, en NNDI. VI. Torino 1960.

${ }^{41}$ CALONGE, La compraventa civil de cosa futura, cit., 33, afirma que la compra del azar se refiere a un doble supuesto.

42 ARANGIO-RUIZ, La compravendita in diritto romano, cit., 119.

43 BESELER, Scritti di diritto romano in onore di Contardo Ferrini (Milano 1946) 3,295. cit., por Arangio-Ruiz, 120 n.1. VASALLI en Studi Giuridici III, 1. (Milano 1970) 372, cit., por Calonge, 34, n.16. 
guna, esta obligación se extendía también al capientem missilia. Es, por tanto, una compra sine condicione, porque lo que se compra es la spes:

\section{D.19,1,11,18 (Ulpianus libro trigésimo secundo ad edictum)}

...veluti cum futurum iactum retis a piscatore emimus aut indaginem plagis positis a venatore, vel pantheram ab aucupe: nam etiamsi nihil capit, nihilo minus emptor pretium praestare necesse habebit...

El jurista Celso (libro vigesimo septimo digestorum) ${ }^{44}$ refiriéndose únicamente al pescador, vendedor, expone dos casos de responsabilidad por dolo, cuando no quiso lanzar la red, en cuyo caso el iudex haría una aestimatio incerti, o cuando no quiso entregar el producto obtenido, y, en consecuencia, el juez realizaría una aestimatio certior $^{45}$. Debemos extender, por analogía, esta misma responsabilidad al auceps, o al capiens missilia, en este último caso, bien porque no actuó diligentemente en la recogida ${ }^{46}$, bien porque no entregó el captus. El hecho de comprar el azar (alea) elimina del vendedor la responsabilidad por evicción.

${ }^{44}$ D. 19.1.12. Si iactum retis emero et iactare retem piscator noluit, incertum eius rei aestimandum est: si quod extraxit piscium reddere mihi noluit, id aestimari debet quod extraxit.

${ }^{45}$ CALONGE, La compraventa civil de cosa futura, cit., 38.

46 FERNÁNDEZ DE BUJÁN, F., Sistema contractual romano, cit., 73.BESELER, Scritti di diritto romano in onore di Contardo Ferrini (Milano 1946) 3,295. cit., por Arangio-Ruiz, 120 n.1. VASALLI en Studi Giuridici III, $1^{\circ}$ (Milano 1970) 372, cit., por Calonge, 34, n.16. 